\title{
Auto-exame das Mamas: Frequêencia do Conhecimento, Prática e Fatores Associados
}

\author{
Breast Self-examination: \\ Frequency of Knowledge, Practice and Associated Factors \\ Ana Paula de Sousa Monteiro, Elizabeth Paiva Pereira Arraes \\ Lucíola de Barros Pontes, Marilia do Socorro dos Santos Campos \\ Rafael Teixeira Ribeiro, Rubens Elísio Brandão Gonçalves
}

\begin{abstract}
RESUM0
Objetivo: estudar a freqüência do conhecimento e prática do auto-exame de mamas (AEM), caracterizando alguns fatores que influenciam sua prática.

Método: durante um mês, foram entrevistadas 505 mulheres atendidas no Centro de Saúde Escola - Marco (CSE-Marco) e no anexo Unidade Materno-Infantil por meio de questionário referente ao conhecimento e prática do AEM e possiveis fatores associados. Verificou-se a correlação entre as variáveis através do teste de $\chi^{2}$.

Resultados: das mulheres entrevistadas, 96,0\% conheciam o AEM. Dentre essas, 58,9\% conheceram-no pela imprensa. Contudo, o meio que proporcionou prática mais correta foi a orientação médica (37,5\%). Apenas $21,8 \%$ das mulheres realizavam o exame mensalmente. O principal motivo da não-realização foi o desconhecimento da técnica (48,2\%). Mulheres entre 30 e 39 anos (30,2\%) apresentaram maior prática mensal do exame e 58,2\% das que o realizavam corretamente tinham pelo menos ensino médio incompleto. Em $58,7 \%$ dos casos, o ginecologista não incentiva a prática do AEM.

Conclusão: o AEM é conhecido por praticamente todas as entrevistadas, embora mais de um terço destas não o realize, principalmente por desconhecimento da técnica. O meio de comunicação que levou a orientação mais eficiente foi a orientação médica, contudo, esta atingiu reduzido número de pacientes. Houve interferência do grau de escolaridade e faixa etária na prática do AEM, não intervindo a presença de casos de câncer na família.
\end{abstract}

PALAVRAS-CHAVE: Auto-exame das mamas. Câncer de mama. Câncer: rastreamento.

\section{Introdução}

O câncer de mama tem sido um dos maiores problemas de saúde pública em todo o mundo, sendo provavelmente o mais temido pelas mulheres devido a sua alta freqüência e pelos seus efeitos psicológicos ${ }^{1}$. Em termos epidemiológicos, o

Universidade do Estado do Pará - Centro de Saúde Escola do Marco e Anexo Unidade Materno Infantil Correspondência:

Lucíola de Barros Pontes

Rua dos Tamoios, $\mathrm{n}^{\circ} 1457$, apto 702

66025-540 - Belém - PA

Batista Campos

Telefone: (91) - 222-3782

e-mail: luciola@interconect.com.br câncer de mama é o tumor de maior incidência em vários países, dentre os quais, o Brasil ${ }^{1}$. Percebe-se que o número de casos novos aumenta cada vez mais e que a projeção desta incidência é ainda maior nos países em desenvolvimento ${ }^{1}$.

Segundo as estimativas do Instituto Nacional do Câncer (INCA) para o ano 2002, 36100 brasileiras receberão o diagnóstico de câncer de mama ${ }^{2}$. No Brasil, ele é responsável por $14,2 \%$ do total de $15,1 \%$ de mortes por neoplasias em mulheres ${ }^{3}$. De acordo com dados do Ministério da Saúde, o câncer de mama ocupa o segundo lugar em casos na região Norte e a terceira colocação no percentual de mortes por câncer no Estado do Pará ${ }^{1-3}$.

Não se pode fazer prevenção primária ao câncer de mama, pois não foi possivel o reconhe- 
cimento de lesões verdadeiramente precurso$\operatorname{ras}^{1,3}$. Assim, a única ação efetiva que se tem é prevenção secundária em termos de diagnóstico em estágios iniciais da doença ${ }^{1}$.

As formas mais eficazes para detecção precoce do câncer de mama são o auto-exame das mamas (AEM), o exame clínico e a mamografia. A mamografia e ultra-sonografia identificam tumores não palpáveis, apresentam alto custo e não fornecem resultados operacionais para serem aplicados em grandes massas populacionais, consagrando-se o AEM mensal como estratégia de escolha, uma vez que se caracteriza como prevenção secundária, sem custos e segura ${ }^{1}$.

As pesquisas indicam impacto significativo do AEM na detecção precoce do câncer de mama, registrando-se tumores primários menores e menor número de linfonodos axilares invadidos pelo tumor (ou por células neoplásicas) nas mulheres que fazem o exame regularmente, além de haver também detecção de pequenas mudanças nas propriedades fisicas das mamas, diminuindo assim a probabilidade de metástase e aumentando a sobrevida. Estudos demonstram que a sobrevida em cinco anos tem sido de $75 \%$ entre as praticantes do AEM contra $57 \%$ entre as não-praticantes ${ }^{4}$.

Assim, objetiva-se estudar a freqüência do conhecimento e prática do AEM, bem como caracterizar alguns fatores que favorecem ou limitam a sua prática, com a finalidade de possibilitar o aperfeiçoamento da assistência prestada à detecção precoce do câncer de mama.

\section{Pacientes e Métodos}

Neste estudo prospectivo, aplicou-se um questionário a mulheres com mais de 20 anos a respeito da freqüência do conhecimento e prática do AEM e possiveis fatores associados. Para a definição da casuística, utilizou-se como critério a faixa etária citada na literatura. A respeito da pergunta "você conhece o auto-exame?", este conhecimento referia-se apenas ao fato de a entrevistada saber ou não da existência do mesmo. Em relação à freqüência da prática do exame, estabeleceram-se, segundo referências do INCA, três parâmetros: a realização mensal, a realização em freqüência não preconizada, ou seja, qualquer intervalo diferente do mensal, e a não-realização. Em termos da realização do exame pelo médico ginecologista e o seu incentivo à prática do autoexame, foram consideradas todas as mulheres entrevistadas que referiram ter ido a pelo menos uma consulta ginecológica.

As entrevistadas faziam parte da demanda espontânea do Centro de Saúde Escola - Marco
(CSE-Marco) e do anexo Unidade Materno-Infantil. Durante o período de um mês (setembro a outubro de 2002), os dados foram colhidos mediante visitas diárias nos períodos da manhã e da tarde, perfazendo um total de 505 entrevistas. As informações foram inseridas em banco de dados do software Epi-Info versão 6.04. As tabelas e gráficos foram construídos no Microsoft Excel 97. A correlação entre as variáveis foi verificada pelo teste $\chi^{2}$, ao nivel de significância $\mathrm{p}=0,05$.

O estudo foi aprovado pela Comissão de Ética da Universidade do Estado do Pará e as entrevistadas forneceram consentimento verbal para participação.

\section{Resultados}

A quase totalidade das entrevistadas $(96,0 \%)$ conhecia o AEM, contudo menos de um terço destas o realizavam corretamente (Tabela 1 ). Dentre as que não o realizavam, o principal motivo foi o desconhecimento da técnica, com 55 mulheres, representando $48,2 \%$ do total (Figura 1 ).

Tabela 1 - Freqüência de realização do auto-exame de mama.

\begin{tabular}{lcr}
\hline Faz o exame com que freqüência? & Freqüência & $\%$ \\
\hline Freqüência não preconizada & 218 & 43,2 \\
Freqüência mensal & 110 & 21,8 \\
Não faz & 177 & 35,0 \\
Total & 505 & 100,0 \\
\hline${ }^{*}<0.05 \chi^{2}$ & &
\end{tabular}

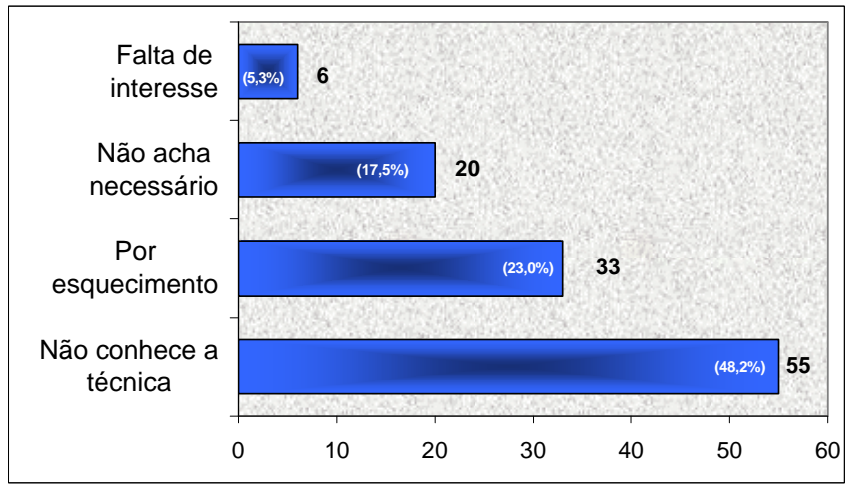

Figura 1 - Principais motivos da não realização do auto-exame da mama (AEM) ${ }^{*} p<0,05\left(\chi^{2}\right)$

A maioria das entrevistadas $(59,0 \%)$ tomou conhecimento do AEM pela imprensa, seguido pelos demais profissionais de saúde $(17,9 \%)$ e médico $(14,8 \%)$, contudo, o meio que levou a prática mais correta foi a orientação médica (Figura 2).

Das pacientes que responderam já ter ido pelo menos uma vez ao ginecologista, $58,7 \%$ refe- 
riram que este não incentiva a prática do AEM, tampouco examina suas mamas $(63,8 \%)$.

Em relação à faixa etária, mulheres entre 30 e 39 anos são as que mais realizam o exame corretamente. A maior parte das mulheres entre 60 e 69 anos não realiza o exame e aquelas que se encontram entre 40 a 49 são as que mais o realizam na freqüência não preconizada (Figura 3).

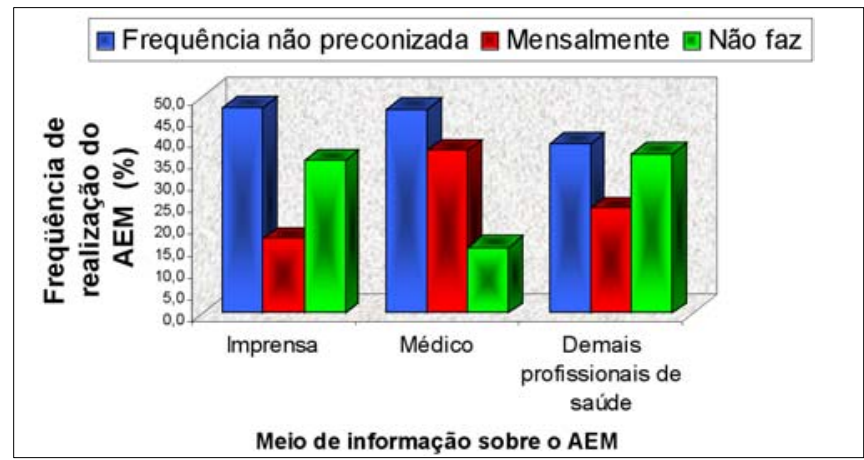

Figura 2 - Freqüência de realização do auto-exame das mamas (AEM) conforme os principais meios de informação ${ }^{*} p<0.05\left(\chi^{2}\right)$

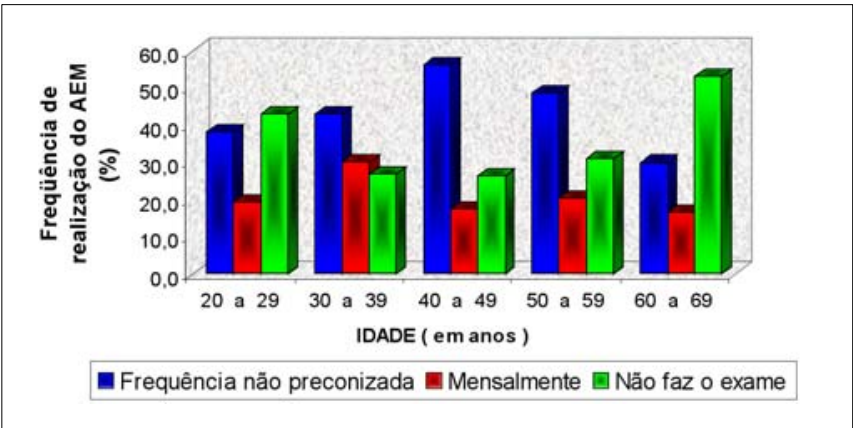

Figura 3 - Freqüência de realização do auto-exame das mamas (AEM) conforme a idade. ${ }^{*} p<0.05\left(\chi^{2}\right)$

Mais de $50 \%$ das entrevistadas que realizavam o exame mensalmente possuíam pelo menos ensino médio incompleto, e daquelas que não o realizavam, mais da metade possuía até o ensino fundamental completo (Figura 4).

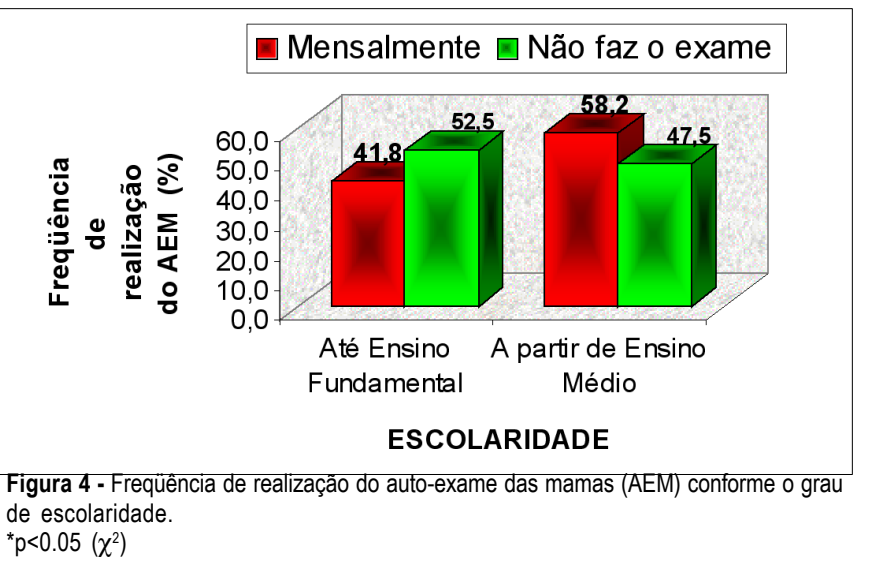

\section{Discussão}

Vários métodos empregados para o diagnóstico precoce do câncer de mama, como a mamografia e ultra-sonografia, que identificam tumores não palpáveis, menores que $1 \mathrm{~cm}$, apresentam alto custo e não fornecem resultados operacionais para serem aplicados em populações. Dessa forma, consagra-se o AEM mensal, inserido num processo educativo, juntamente com o exame das mamas por profissional treinado, como estratégia de escolha ${ }^{5}$. Por isso, tornou-se importante estudar a freqüência do conhecimento e prática do AEM.

Em relação ao grau de conhecimento e prática correta do AEM, os resultados obtidos ratificaram o estudo de Freitas Júnior et al. ${ }^{6}$, segundo o qual apenas um terço das mulheres que conheciam o AEM o realizavam de forma sistemática.

A freqüência de realização do auto-exame influencia diretamente a acurácia do mesmo ${ }^{7}$. Segundo Laganá et al. ${ }^{5}$, para mulheres que nunca praticaram o AEM, geralmente os nódulos cancerígenos identificados medem $3,5 \mathrm{~cm}$, para as que o praticam eventualmente, os nódulos têm cerca de 2,5 cm; e, para as que o fazem mensalmente, são identificados com aproximadamente $2 \mathrm{~cm}$ ou menos. Aquelas que praticam o auto-exame e descobrem nódulos, têm expectativa de vida de $75 \%$ e as que não o fazem reduzem suas chances para $59 \%$.

Constatou-se que a maioria das entrevistadas realizava o AEM em freqüência não preconizada e mais de um terço não o realizava. Destas, a grande maioria conhecia o AEM, sendo que o principal motivo da não-realização foi o desconhecimento da técnica, seguido por esquecimento, conforme assinalado por Caetano e Helene ${ }^{8}$, e o que se justifica pelo fato de que a maioria dos serviços que realizam atendimento em saúde da mulher apresentam propostas que não são acompanhadas de treinamento adequando para a prática do $\mathrm{AEM}^{9-11}$. É importante notar que dentre os motivos da não-realização, encontraram-se justificativas como "não achar necessário" e "falta de interesse", demonstrando que apenas transmitir a informação não é suficiente para a mudança de comportamento, já que a prática do AEM depende da decisão da cliente, a partir da compreensão e interpretação que tem da possibilidade de prevenir e ser responsável pela sua própria saúde ${ }^{8}$.

Laganá e Imanische ${ }^{9-11}$ também afirmam ser o serviço de saúde e os meios de comunicação em massa os maiores disseminadores do conhecimento e ensino da prática do AEM, conforme assinalado neste estudo. A orientação pela imprensa atingiu grande número de mulheres, porém, a infor- 
mação fornecida não se mostrou eficiente por não ensinar a prática correta do exame, visto que grande parte delas realizavam o exame com freqüência incorreta, o que diminui sua acurácia, ou não realizavam-no. Desse modo, as campanhas que atingem a maior parte da população não orientam a prática de forma adequada, além de não haver programas eficientes de ensino do AEM em serviços de atendimento à mulher. É importante que a detecção precoce do câncer de mama por meio do ensino do auto-exame seja de responsabilidade de todos os que assistem pacientes do sexo feminino, e não apenas daqueles que atuam em programas especificos para esse fim $^{12}$.

A orientação médica se mostrou a mais eficiente ao proporcionar o maior percentual de prática correta, indicando, assim, a boa qualidade da orientação, apesar de ter atingido pequeno número de pacientes. Isso é fato preocupante, pois já existem estudos que começam a questionar a confiabilidade do AEM, como o desenvolvido pelo Centro de Pesquisas em Câncer Fred Hutchinson, de acordo com o qual o AEM não é útil para a detecção precoce de tumores malignos nem ajuda a diminuir a mortalidade da doença ${ }^{13}$, não sendo método de escolha para o diagnóstico precoce, mas sim método complementar que desperte a atenção da mulher para o auto-cuidado, fazendo-a perceber qualquer alteração e, com isso, procurar um profissional. Há, portanto, tendência a se valorizar o bom exame clínico das mamas pelo médico e a solicitação de possivieis exames para detecção de tumores, necessitando-se de mudança de comportamento deste profissional e conscientização de seu papel importante neste contexto.

Em relação à idade, achado positivo foi o fato de que as mulheres que mais realizam o exame corretamente ou com freqüência preconizada localizam-se principalmente na faixa etária de 35 a 49 anos, em que há maior incidência da doença, o que pode proporcionar diagnóstico precoce, importante num período em que a mulher é economicamente ativa e pode estar constituindo família. $\mathrm{O}$ fato de as mulheres idosas não realizarem o exame é explicado por terem dificuldades como: diminuição do tato, da visão e da sensibilidade; aumento do pudor em se despir e se tocar, e maior incidência de osteoartrite ${ }^{6}$. Além disso, mulheres nessa faixa etária são menos receptivas a novas informações e já não apresentam auto-cuidado tão pronunciado com as mamas, associando isto ao fato de terem entrado na menopausa. Notou-se, ainda, que quase metade das mulheres jovens (20 a 29 anos) também não realizam o auto-exame, fato atribuído à idéia de que pela pouca idade não há necessidade de prevenção nem risco de apresentar a doença.
O estudo demonstrou que o grau de escolaridade influencia na prática do $\mathrm{AEM}$, concordando com o achado de Freitas Júnior et $a .^{6}{ }^{6}$, em que o grupo de mulheres com melhor nivel educacional é o que tem maior acesso ao conhecimento do AEM e, por conseguinte, o que mais o pratica.

Nos países em desenvolvimento, como no caso do Brasil, não há política adequada visando ao diagnóstico precoce do câncer de mama e por mais um longo período, provavelmente, não haverá condições ideais de lançar campanha com custos elevados. Ao mesmo tempo, nesses países, não há investimento em educação pública, o que dificulta o acesso à informação. Fato notado durante as entrevistas foi a vinculação do termo "auto-exame" à mamografia, fazendo com que as pacientes comumente confundissem os dois tipos de exames, muitas vezes havendo necessidade de fazer distinção entre ambos para que fosse dada continuidade à entrevista. Desta forma, torna-se de fundamental importância o conhecimento de quais os grupos populacionais que necessitam de maior atenção e, a partir daí, identificar qual seria o melhor método de alcançá-los para a difusão da prática do auto-exame ${ }^{6}$.

A presença de histórico familiar de câncer de mama não interferiu na sua prática, conforme constatado por Freitas Júnior et al. ${ }^{14}$. A presença de casos de câncer não significa um fator de maior auto-cuidado, evidenciado-se a distância entre a possibilidade de ocorrência do câncer e sua percepção, o que pode estar relacionado ao medo e à mística do câncer, seu tratamento e morte ${ }^{6}$.

Para que a prática do AEM consiga alcançar seu objetivo de detecção precoce do câncer e conseqüente queda da mortalidade, as campanhas sobre o mesmo devem ser realizadas de modo a fornecer informações mais completas sobre a técnica e a importância do auto-cuidado, concomitante ao incentivo na área educativa, para que essas informações se incorporem ao comportamento da mulher. A divulgação do método deve ser estimulada em todos os niveis assistenciais, tanto por médicos quanto por demais profissionais de saúde, ressaltando-se a sua importância dentro do contexto assistencial ao sexo feminino, para que sejam alcançados os diferentes grupos sociais de forma efetiva.

\section{ABSTRACT}

Purpose: to study frequency of the knowledge and practice of breast self-examination (BSE), characterizing some factors that may interfere in its practice.

Method: during one month, 505 women, who were patients 
of the "Centro de Saúde Escola-Marco (Belém-Pará)", were interviewed, answering questions about the practice and knowledge on breast self-examination and associated factors. Statistical analysis was perfoprmed using the $\chi^{2}$ test. Results: $96 \%$ of the women knew about BSE. Among these, $58.9 \%$ learned about it via the midia. However, the way that provided the most correct practice was medical orientation (37.5\%). Only $21.8 \%$ of the women performed the examination monthly. The main reason for the low adhesion was unfamiliarity with the technique (48.2\%). Women between 30 and 39 years presented the highest percentage of monthly practice (30.2\%). The women (58.2\%) who carried out the monthly examination had at least an incomplete high school degree. In $58.7 \%$ of the cases, the gynecologist did not stimulate the practice of the BSE.

Conclusions: although BSE is known by practically all the interviewees, more than one third of them do not practice it. The main reason of the low adhesion was the unfamiliarity with the technique. Level of instruction and age of the women interfered in the practice of BSE, but presence of breast cancer cases in the family did not.

KEYWORDS: Breast self-examination. Breast cancer. Early diagnosis.

\section{Agradecimentos}

À Marizeth Carvalho de Andrade e à Prof. ${ }^{a}$ Dr. $^{a}$ Lúcia Maria Frazão Helene.

\section{Referências}

1. Gonçalves SMCM, Dias MR. A prática do auto-exame da mama em mulheres de baixa renda: um estudo de crenças. Estud Psicol 1999; 4:141-59.

2. INCA. Estimativa de incidência e mortalidade por câncer no Brasil. [online]. Rio de Janeiro; 2002 [acesso em 2002]. Disponivel em: http:// www.inca.gov.br/cancer/epidemiologia/ estimativa2002/estimativas.html

3. Cascaes APR, Pinho ALG, Neiva Neto EC, Santos HL, Costa JBG, Nascimento LL. As principais causas da não realização do auto-exame das mamas na Unidade Básica de Saúde da Pedreira. Anais do $3^{\circ}$ Encontro Regional dos Estudantes de Medicina; maio 2002; Belém, Brasil; 2002. p.5.
4. Ludwick R. Breast examination in the older adult. Cancer Nurs 1988; 11:99-102.

5. Laganá MTC, Gualda DMR, Hashimoto MRH, Imanichi RM. Auto exame de mama: identificação dos conhecimentos, atitudes, habilidades e práticas (CAHP) requeridos para elaboração de propostas educativas. Rev Esc Enfermagem USP 1990; 24:281-99.

6. Freitas Júnior R, Soares VF, Melo NF, Andrade ML, Philocréon GR. Fatores determinantes do conhecimento e prática do auto-exame de mama. Rev Bras Ginecol Obstet 1996; 18:387-91.

7. Borba AA, Souza RM, Lazzaron AR, Defferrari R, Scherer L, Frasson AL. Freqüência de realização e acurácia do auto-exame das mamas na detecção de nódulos em mulheres submetidas à mamografia. Rev Bras Ginecol Obstet 1998; 20:37-43.

8. Caetano VC, Helene LMFH. Auto-exame das mamas: um estudo das mulheres que ocupam o território da Unidade de Saúde Ambulatorial Qualis. Anais do $7^{\circ}$ Congresso Paulista de Saúde Pública; 2001. Santos, Brasil. São Paulo: Associação Paulista de Saúde Pública; 2001. p.120.

9. Laganá MTC, Imanische RM. Câncer de mama feminina e auto-exame da mama: identificação de fatores de risco numa população de mulheres atendidas em unidade básica de saúde. Parte i. Âmbito Hosp 1991; 3:19-23.

10.Laganá MTC, Imanische RM. Câncer de mama feminina e auto-exame da mama: identificação de fatores de risco numa população de mulheres atendidas em unidade básica de saúde. Parte ii. Âmbito Hosp 1991; 3:4-6.

11.Laganá MTC, Imanische RM. Câncer de mama feminina e auto-exame da mama: identificação de fatores de risco numa população de mulheres atendidas em unidade básica de saúde. Parte iii. Âmbito Hosp 1991; 3:7-10.

12.Hood MD, Vargens OMC. Prevenção do câncer de mama: somos todos responsáveis. Rev Enfermagem UERJ 1995; 3:108-10.

13.Thomas DB, Gao DL, Self SG, et al. Randomized trial of breast self-examination in Shanghai: methodology and preliminary results. J Natl Cancer Inst 1997; 89:355-65.

14.Freitas Júnior R, Baêta LF, Aires NM, Paulinelli RR, Finotti MCF, Silveira MT. Auto-exame das mamas entre estudantes de medicina. Rev Bras Ginecol Obstet 1999; 21:287-90. 